\title{
Method of evaluating energy released by a warhead charge based on average fragment quality
}

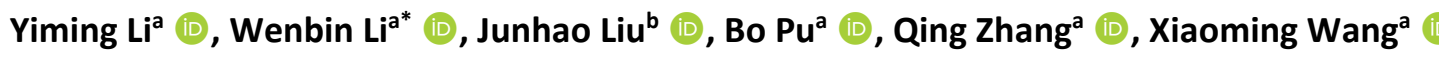 \\ a Ministerial Key Laboratory of ZNDY, Nanjing University of Science and Technology, Nanjing 210094, China. E-mail: \\ Liyiming0919@163.com, Iwb2000cn@njust.edu.cn, 392153931@qq.com, qingzhang827@163.com, 202xm@163.com \\ b Systems Engineering Research Institute, China State Shipbuliding Corporation Limited, Beijing 100070, China. \\ E-mail: 1009797882@qq.com \\ * Corresponding author
}

https://doi.org/10.1590/1679-78256481

\begin{abstract}
Limited initiation energy or external stimulation may cause incomplete detonation in a warhead. At present, there is no quantitative method to characterize the energy release of a warhead charge for incomplete detonation such as explosion and deflagration. We propose a method based on average fragment quality to characterize the energy released by a warhead charge. The theoretical study shows that the average fragment quality after warhead initiation is inversely proportional to the initial fragment velocity. The relationship between the average fragment quality and explosive energy release is established and verified by experiment. This relation can be used to determine the charge energy released after warhead initiation. It provides a theoretical basis for optimizing efficiency of charge energy use in high-energy conventional damage technology and warhead design, and provides a quantitative method for evaluating insensitive ammunition.
\end{abstract}

\section{Keywords}

Ammunition; Natural fragments; Average quality; Explosive energy release

\section{Graphical Abstract}
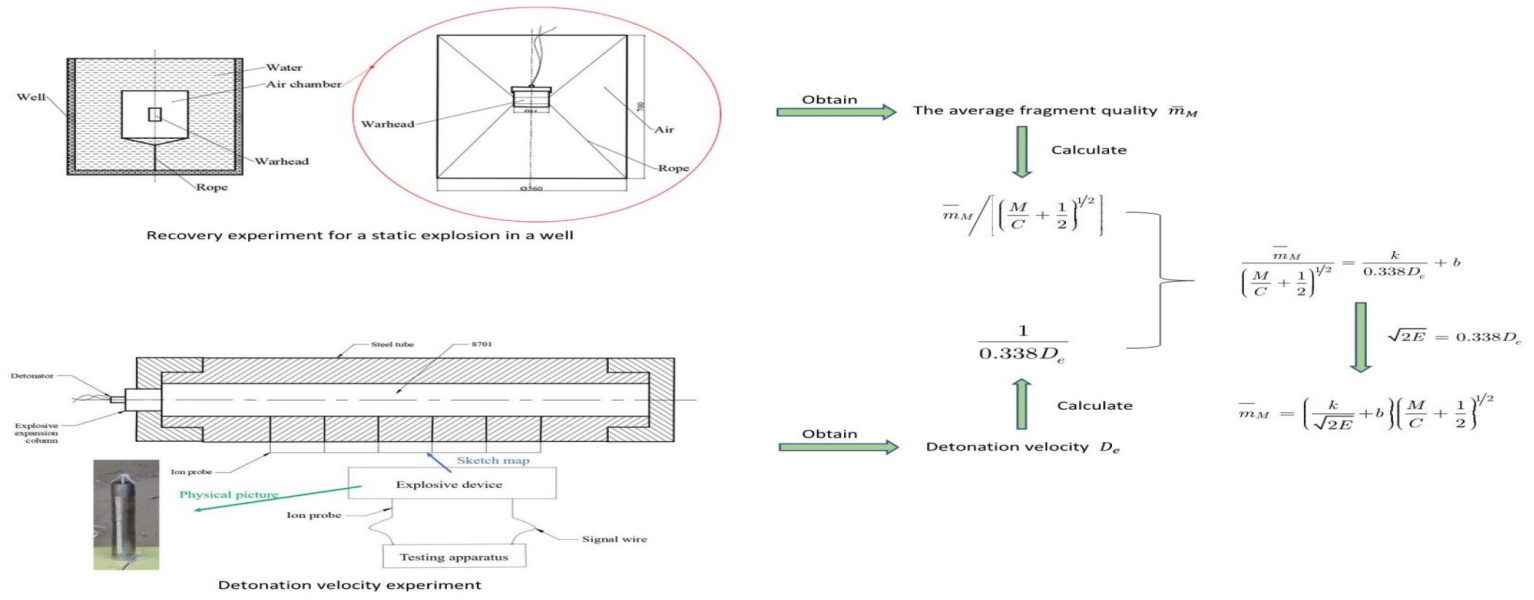

Received March 11, 2021. In revised form March 29, 2021. Accepted March 29, 2021. Available online March 30, 2021. https://doi.org/10.1590/1679-78256481

(C) Latin American Journal of Solids and Structures. ISSN 1679-7825. Copyright @ 2021. This is an Open Access article distributed under the terms of the Creative Commons Attribution License, which permits unrestricted use, distribution, and reproduction in any medium, provided the original work is properly cited. 


\section{INTRODUCTION}

Limited initiation energy or external stimulation may cause incomplete detonation in a warhead charge. Complete warhead detonation has been fully studied (Mott and Linfoot, 1943; Mott, 1947; Grisaro and Dancygier, 2016; Held, 1990), but less study has been done on quantitatively characterizing charge energy release for incomplete detonation such as explosion and deflagration. This makes it difficult to evaluate warhead charge initiation and detonation ability. This is especially true for the insensitive ammunition developed in recent years, for which the reaction degree such as deflagration and explosion can only be evaluated qualitatively, not quantitatively.

Smirnov et al. (2016) demonstrated the relation between the local curvature of the detonation front and the detonation velocity, and derived the equation of the detonation front shape to describe non-ideal detonation of condensed high explosives. Shikha Gupta et al. (2015) used linear discriminant analysis and k-means clustering to discriminate between ideal, non-ideal, and non-explosive. Song et al. (2011) obtained the pressure-volume relation for non-ideal detonation by modifying the equation of state under the $\mathrm{C}-\mathrm{J}$ model, and discussed the energy use of non-ideal detonation expansion under different energy release rates and energy release indexes. However, their paper only does a theoretical analysis and obtains a qualitative description whose results cannot be applied to an actual test.

Gurney (1943) studied the fragment motion caused by explosive detonation and obtained the energy use of an explosive by calculating the fragment velocity. Although the fragment velocity reflects the ability of the explosive to drive the object to some extent, the fragment velocity is assumed from the plane structure. This method does not consider the different fragment velocity of warhead formation. Therefore, a single velocity measurement cannot well determine the detonation energy in an actual test.

In this study, the initial velocity $v_{0}$ is used to establish the relationship between the average quality $\bar{m}_{M}$ of fragments formed after warhead initiation and the explosive energy release $E$. This yields a quantitative method to evaluate the explosive energy release after warhead initiation using the average quality of natural fragments.

\section{Theoretical analysis}

Zhu et al. (2019) showed that:

$$
\bar{m}_{M}=k^{*} \rho \delta a_{x} b_{x}
$$

where $\bar{m}_{M}$ is the average fragment quality; $k^{*}$ is the proportionality coefficient of the fragment mass; $\rho$ is the shell density; $\delta$ is the average fragment thickness; $a_{x}$ is the fragment width, which reflects the axial position of the shell; and $b_{x}$ is the fragment length. In addition,

$$
\begin{aligned}
& a_{x}=\left[\frac{6 \sqrt{2} \Gamma\left(r_{0}+R_{0}\right)^{2}}{\rho v_{0}{ }^{2}}\right]^{1 / 3} \\
& b_{x}=a_{x}{ }^{2}\left[\frac{\rho v_{0} u}{\left(r_{0}+R_{0}\right) / 2 \sigma_{s}}\right] F(x)^{\lambda} \\
& \delta=R_{0}-r_{0}
\end{aligned}
$$

where $\Gamma$ is the fracture energy of the shell at the unit area, $r_{0}$ and $R_{0}$ are the initial inner and outer radii of the shell, $v_{0}$ is the velocity of each cylindrical microelement, $u$ is the velocity with which each crack extends, $\sigma_{s}$ is the fracture strength, $F(x)$ is the correction function, and $\lambda$ denotes a constant.

According to equations (1)-(4), the average fragment quality $\bar{m}_{M}$ is inversely proportional to $v_{0}$, which can be obtained from the Gurney equation (Gurney, 1943), 


$$
v_{0}=\sqrt{2 E}\left(\frac{M}{C}+\frac{1}{2}\right)^{-\frac{1}{2}}
$$

where $C$ is the explosive mass, $M$ is the shell mass, $E$ is the energy released by the explosive, and $\sqrt{2 E}$ is the Gurney constant of the explosive. According to Huang and Zu (2014),

$$
\sqrt{2 E}=0.338 D_{e}
$$

where $D_{e}$ is the detonation wave velocity. Thus,

$$
\bar{m}_{M} \propto \frac{1}{0.338 D_{e}}\left(\frac{M}{C}+\frac{1}{2}\right)^{1 / 2}
$$

More precisely,

$$
\frac{\bar{m}_{M}}{\left(\frac{M}{C}+\frac{1}{2}\right)^{1 / 2}}=\frac{k}{0.338 D_{e}}+b
$$

According to equation (6), equation (8) can be written as

$$
\frac{\bar{m}_{M}}{\left(\frac{M}{C}+\frac{1}{2}\right)^{1 / 2}}=\frac{k}{\sqrt{2 E}}+b
$$

\section{Rearranging gives}

$$
\bar{m}_{M}=\left(\frac{k}{\sqrt{2 E}}+b\right)\left(\frac{M}{C}+\frac{1}{2}\right)^{1 / 2}
$$

\section{Determination of the calculation formula}

To use equation (9), it is necessary to determine the values of $k$ and $b$. Given a warhead structure, the values of $\bar{m}_{M}$ and $D_{e}$ after complete detonation are calculated and then $k$ and $b$ can be obtained from linear fitting.

Steel 1045 with a wall thickness of $7 \mathrm{~mm}$ is selected for the warhead shell, and the shell density $\rho$ is $7.85 \mathrm{~g} / \mathrm{cm}^{3}$. The charge size is $70 \mathrm{~mm} \times 70 \mathrm{~mm}$, and the charge type is TNT. When the charge size is kept unchanged and the charge density is changed, as shown in Table 1, the detonation velocity changes with the charge density according to (Dobratz and Crawford, 1985)

$$
D_{e}=A+B \rho_{e}
$$

where $\rho_{e}$ is the charge density, and $A$ and $B$ are related to the type of explosive. When the explosive is TNT, $\rho_{e} \in 0.57-$ $1.66 \mathrm{~g} / \mathrm{cm}^{3}, A=1.844 \mathrm{~km} / \mathrm{s}$, and $B=3.204 \mathrm{~km} / \mathrm{s}\left(\mathrm{cm}^{3} / \mathrm{g}\right)$.

If the charge is a mixed explosive, the detonation velocity can also be calculated according to (Dobratz and Crawford, 1985) 
$D_{e}=\frac{D_{e \max }}{4}+\frac{3 D_{e \max }}{4 \rho_{e \max }} \rho_{e}$

where $D_{e}$ is the detonation velocity when the charge density is $\rho_{e}, D_{e \max }$ is the theoretical detonation velocity of a mixed explosive, and $\rho_{e \max }$ is the theoretical density of a mixed explosive.

The software Damage Assessment Software, independently developed by ZNDY of Minimal Key Laboratory (Zhu et al., 2019; Zhu et al., 2018), is used to calculate average fragment quality greater than $1 \mathrm{~g}$ when explosives with different charge densities are completely detonated. The software uses a micro-cylinder model of a warhead to analyze shell fragmentation at different axial positions when one end is initiated. The existing theory is used to establish a simplified model of the average mass of fragments formed at different axial positions of the shell. The calculated parameters are shown in Table 1.

Table 1: Charging schemes and calculated parameters.

\begin{tabular}{cccc}
\hline$\rho_{e}\left(\mathbf{g} / \mathbf{c m}^{\mathbf{3}}\right)$ & $D_{e} \mathbf{( k m / s )}$ & $\bar{m}_{M} \mathbf{~ ( g )}\left(\frac{M}{C}+\frac{1}{2}\right)^{1 / 2}$ \\
\hline 1.66 & 7.1626 & 2.86 & 1.598 \\
1.64 & 7.0986 & 2.89 & 1.606 \\
1.60 & 6.9704 & 2.91 & 1.622 \\
1.50 & 6.6500 & 3.15 & 1.665 \\
1.40 & 6.3296 & 3.29 & 1.713 \\
1.20 & 5.6888 & 3.74 & 1.828 \\
1.00 & 5.0480 & 4.26 & 1.977 \\
\hline
\end{tabular}

Figure 1 is the plot of the relationship between $\bar{m}_{M} /\left[\left(\frac{M}{C}+\frac{1}{2}\right)^{1 / 2}\right]$ and $\frac{1}{0.338 D_{e}}$ according to the data in Table 1.

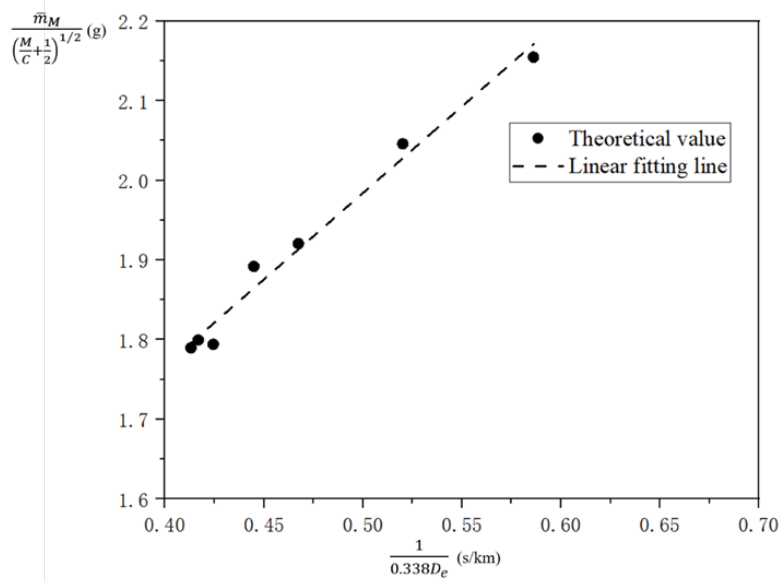

Figure 1: Relationship between average fragment quality and released energy of TNT.

The following equation is obtained by linear fitting in Figure 1:

$\frac{\bar{m}_{M}}{\left(\frac{M}{C}+\frac{1}{2}\right)^{1 / 2}}=\frac{2.17271}{0.338 D_{e}}+0.89796$

The coefficient of determination $R^{2}=0.98218$.

Substituting equation (6) into equation (12) yields 


$$
\bar{m}_{M}=\left(\frac{2.17271}{\sqrt{2 E}}+0.89796\right)\left(\frac{M}{C}+\frac{1}{2}\right)^{1 / 2}
$$

The above method is used to calculate the values of $k$ and $b$ in equation (9) for different charge types, and the results are shown in Table 2. It is seen from Table 2 that $k$ and $b$ are related to the type of explosive.

Table 2: $k$ and $b$ values of different charge types.

\begin{tabular}{|c|c|c|c|c|}
\hline \multirow{2}{*}{ Types of explosives } & $A(\mathrm{~km} / \mathrm{s})$ & $B \mathrm{~km} / \mathrm{s}\left(\mathrm{cm}^{3} / \mathrm{g}\right)$ & \multirow{2}{*}{$k$} & \multirow{2}{*}{$\boldsymbol{b}$} \\
\hline & (Dobratz and Crawford, 1985) & (Dobratz and Crawford, 1985) & & \\
\hline TNT & 1.844 & 3.204 & 2.17271 & 0.89796 \\
\hline RDX & 2.395 & 2.589 & 1.43455 & 0.92177 \\
\hline RDX/TNT (60/40) & 2.805 & 3.002 & 3.15857 & 0.48902 \\
\hline
\end{tabular}

\section{Validation of the proposed formula}

To verify the applicability of equation (13), a recovery experiment was designed for a static explosion in a well using 8701 explosive. Warhead fragments with different densities were recovered, and the average fragment quality $\bar{m}_{M}$ was obtained when the warhead was completely detonated. An ion probe (Zheng et al.,1990) was used to measure the detonation velocities of fragments with different densities. Then we obtained the relationship between the average fragment quality $\bar{m}_{M}$ and the released energy of 8701 explosive.

\subsection{Recovery experiment for a static explosion in a well}

The research objects were steel 1045 and 8701 high explosive. The steel 1045 cylinder shell had a density of $7.83 \mathrm{~g} / \mathrm{cm}^{3}$. The shell thickness was $7 \mathrm{~mm}$, and the mass was about $816.3 \mathrm{~g}$. The size of the 8701 high explosive was kept at $\Phi 70 \mathrm{~mm} \times 61 \mathrm{~mm}$ while the charge density was changed. Four different charge densities were selected, as shown in Table 3. The detonation point was at the center of one end of the warhead where, to fix and locate the detonator, a cover was placed around and over the end cover on the detonation end. To complete detonation of the 8701 high explosive, desensitized RDX with a size of $\Phi 20 \mathrm{~mm} \times 20 \mathrm{~mm}$ was used as the booster explosive. Figure 2 shows each part of the warhead and the assembled warhead.

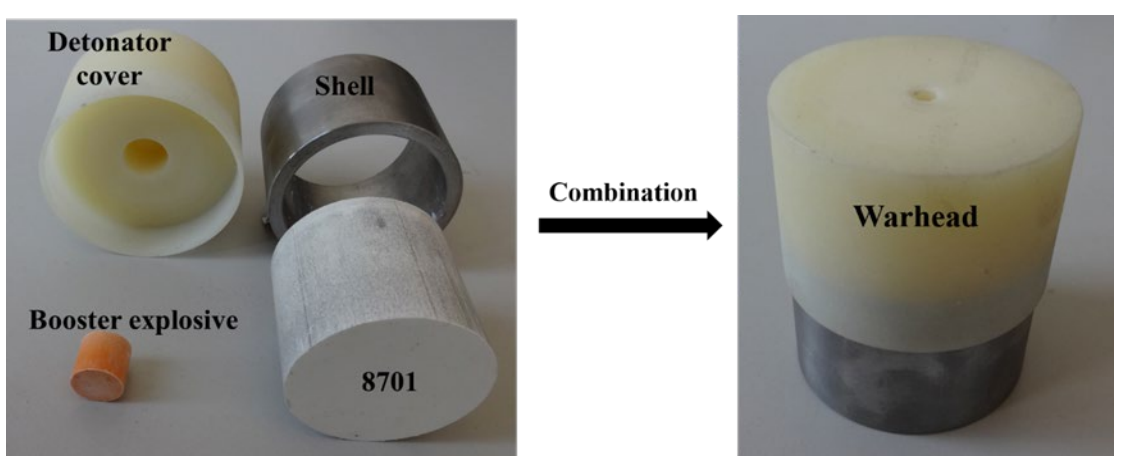

Figure 2: Warhead structure for the recovery experiment for a static explosion in a well

Table 3: Schemes of the recovery experiment for a static explosion in a well.

\begin{tabular}{cccccc}
\hline No. & \#1 & \#2 & \#3 & \#4 \\
\hline$\rho_{e}\left(\mathrm{~g} / \mathrm{cm}^{3}\right)$ & 1.09 & 1.30 & 1.54 & 1.67 \\
\hline
\end{tabular}

The length and internal diameter of the well were $10 \mathrm{~m}$ and $8 \mathrm{~m}$. The warhead was placed in the middle of the air chamber. To prevent the water in the well from entering the air chamber before the warhead exploded, a plastic waterproof sleeve was added outside the air chamber. The air chamber was placed in the middle of the well, as shown in Figure 3. A $0.1 \mathrm{~mm} \times 0.1 \mathrm{~mm}$ multilayer nylon mesh was used to recover fragments. After the experiment, the fragments were removed from the air chamber and dried. The fragments were weighed with a balance with an accuracy of $0.01 \mathrm{~g}$. 

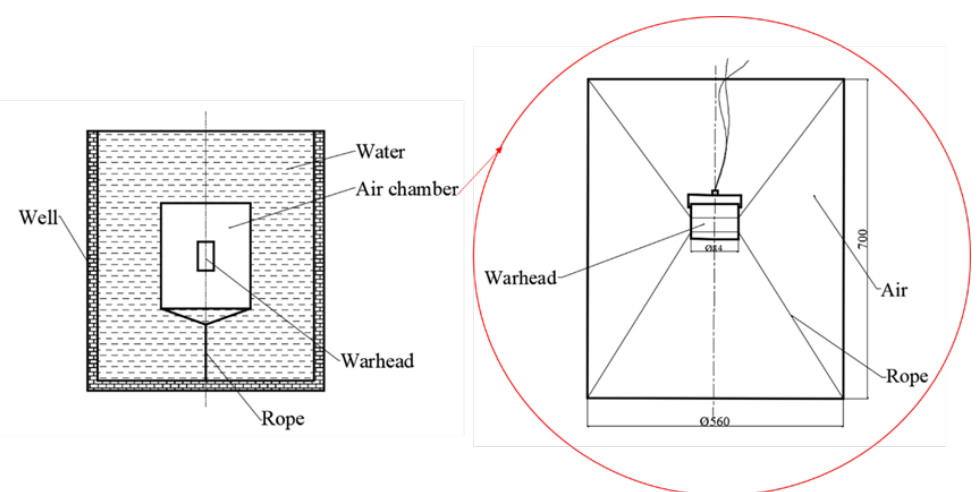

Figure 3: Schematic of the recovery experiment for a static explosion in a well.

After the recovered fragments were weighed and counted, the average fragment qualities greater than $1 \mathrm{~g}$ were obtained. The fragments recovered from the experiment are shown in Figure 4. The statistical experimental results are shown in Table 4. If $M(\mathrm{~g})$ is defined as the shell quality, $M_{0}(\mathrm{~g})$ as the total quality of fragments recovered, and $\gamma$ as the fragment recovery rate, then $\gamma=M_{0} / M \times 100 \%$. If $N$ is the total number of fragments with mass greater than $1 \mathrm{~g}, M_{1}$ (g) the total mass of fragments with mass greater than $1 \mathrm{~g}$, and $\bar{m}_{M}(\mathrm{~g})$ the average fragment quality greater than $1 \mathrm{~g}$, then $\bar{m}_{M}=M_{1} / N \times 100 \%$.
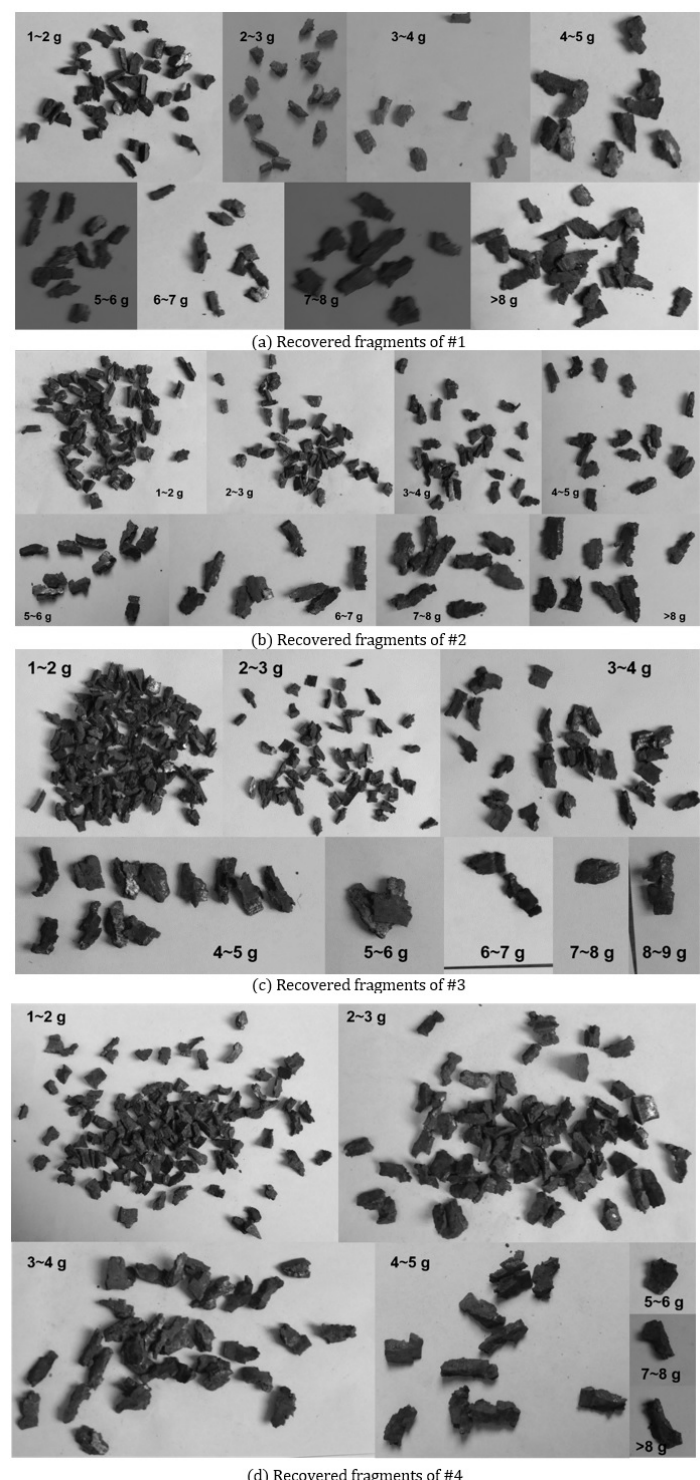

Figure 4: Fragments recovered from a static explosion in a well. 
Table 4: Statistical results from the recovery experiment for a static explosion in well.

\begin{tabular}{ccccccc}
\hline No. & $\boldsymbol{M}(\mathbf{g})$ & $\boldsymbol{M}_{\mathbf{0}}(\mathbf{g})$ & $\boldsymbol{\gamma} / \%$ & $\boldsymbol{N}$ & $\boldsymbol{M}_{\mathbf{1}}(\mathbf{g})$ & 655.14 \\
\hline$\# 1$ & 816.3 & 743.84 & 91.12 & 128 & $\overline{\boldsymbol{m}}_{\boldsymbol{M}}(\mathbf{g})$ & 5.1183 \\
$\# 2$ & 818.6 & 767.09 & 93.71 & 198 & 640.64 & 3.2356 \\
$\# 3$ & 815.9 & 727.12 & 89.12 & 231 & 524.05 & 2.2686 \\
$\# 4$ & 816.7 & 749.05 & 91.72 & 247 & 552.44 & 2.2366 \\
\hline
\end{tabular}

The software Damage Assessment Software was used to model the warhead used in the recovery experiment for the static explosion in the well. We calculated the average of all fragment qualities $\left(\bar{m}_{M}\right)$ greater than $1 \mathrm{~g}$ produced by 8701 explosive in complete detonation under the different charge densities. The calculation results are shown in Table 5. The error between the experimental and calculated values is less than $10 \%$, which shows that the software is reliable in calculating the average fragment quality $\bar{m}_{M}$.

Table 5: Experimental and calculated values of the average of all fragment qualities $\left(\bar{m}_{M}\right)$ greater than $1 \mathrm{~g}$.

\begin{tabular}{cccc}
\hline$\rho_{e}\left(\mathrm{~g} / \mathrm{cm}^{3}\right)$ & Experimental value $(\mathrm{g})$ & Calculated value $(\mathrm{g})$ & Error $/ \%$ \\
\hline 1.09 & 5.1183 & 4.6775 & 8.61 \\
1.30 & 3.2356 & 3.4588 & 6.90 \\
1.54 & 2.2686 & 2.4828 & 9.44 \\
1.67 & 2.2366 & 2.4586 & 9.92 \\
\hline
\end{tabular}

\subsection{Detonation velocity experiment}

A TSN632M 32-channel detonation velocity meter was used for the detonation velocity experiment. The densities of the 8701 explosives were $1.09 \mathrm{~g} / \mathrm{cm}^{3}, 1.30 \mathrm{~g} / \mathrm{cm}^{3}, 1.54 \mathrm{~g} / \mathrm{cm}^{3}$, and $1.67 \mathrm{~g} / \mathrm{cm}^{3}$, and the sizes were all $\Phi 30 \mathrm{~mm} \times$ $270 \mathrm{~mm}$ with cylindrical charges. The size of the explosive expansion column was $\Phi 20 \mathrm{~mm} \times 20 \mathrm{~mm}$, and a JH-14 charge with a \#8 detonator hole was at one end. A \#8 electric detonator was used to detonate at that end. The schematic of the experimental device is shown in Figure 5.

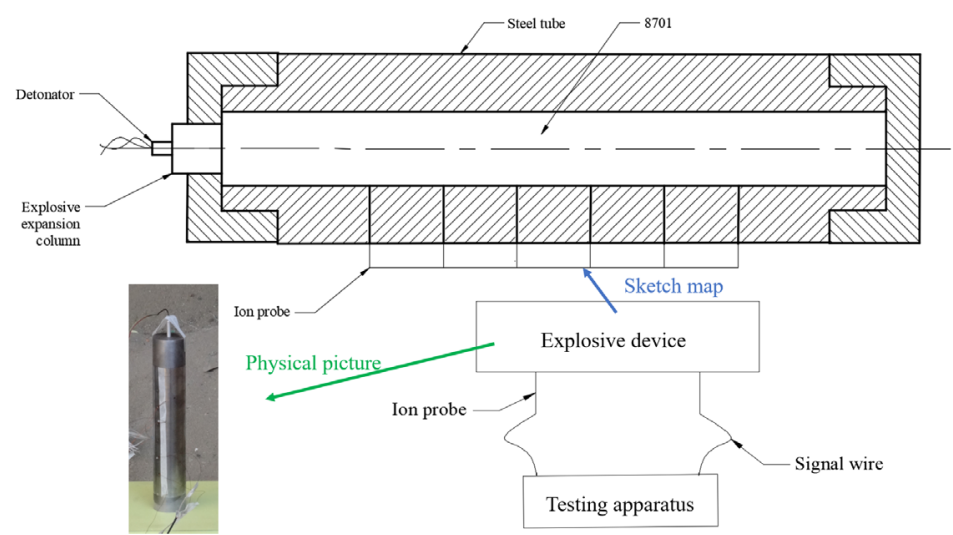

Figure 5: Schematic of the device for the detonation velocity experiment.

An ion probe was used to measure the detonation velocities of 8701 explosive with different charge densities, as shown in Table 6 . The detonation velocity can also be calculated according to equation (11), where $D_{\text {emax }}=8425 \mathrm{~m} / \mathrm{s}$ and $\rho_{\text {emax }}=1.69 \mathrm{~g} / \mathrm{cm}^{3}$. The error between the experimental and calculated values is less than $5 \%$, which indicates the reliability of equation (11).

Table 6: Detonation velocities of 8701 explosive with different charge densities.

\begin{tabular}{ccccc}
\hline No. & $\boldsymbol{\rho}_{\boldsymbol{e}}\left(\mathrm{g} / \mathrm{cm}^{\mathbf{3}}\right)$ & Experimental value $(\mathbf{k m} / \mathbf{s})$ & Calculated value $(\mathbf{k m} / \mathbf{s})$ & Error $/ \%$ \\
\hline$\# 5$ & 1.09 & 5.94265 & 6.18165 & 4.02 \\
$\# 6$ & 1.30 & 6.79663 & 6.96683 & 2.50 \\
$\# 7$ & 1.54 & 7.55984 & 7.86416 & 4.03 \\
$\# 8$ & 1.67 & 8.11254 & 8.35022 & 2.93 \\
\hline
\end{tabular}


Fig. 6 is the plot of the relationship between $\bar{m}_{M} /\left[\left(\frac{M}{C}+\frac{1}{2}\right)^{1 / 2}\right]$ and $\frac{1}{0.338 D_{e}}$ according to the data in Tables 5 and 6 .

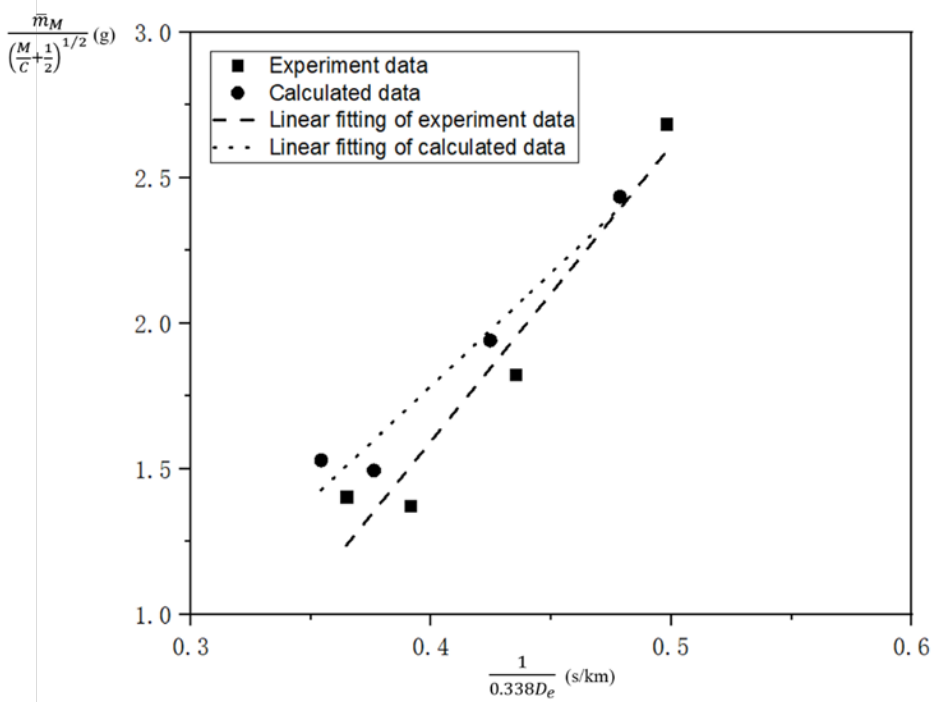

Figure 6: Relationship between the average fragment quality and the released energy of 8701 explosive.

Linear fitting of the experimental data in Fig. 6 yields

$\frac{\bar{m}_{M}}{\left(\frac{M}{C}+\frac{1}{2}\right)^{1 / 2}}=\frac{10.15824}{0.338 D_{e}}-2.46798$

The coefficient of determination $R^{2}=0.93545$. Rearranging gives equation (14), we get

$\bar{m}_{M}=\left(\frac{10.15824}{\sqrt{2 E}}-2.46798\right)\left(\frac{M}{C}+\frac{1}{2}\right)^{1 / 2}$

Fitting the calculated data in Fig. 6 yields

$\frac{\bar{m}_{M}}{\left(\frac{M}{C}+\frac{1}{2}\right)^{1 / 2}}=\frac{7.7857}{0.338 D_{e}}-1.32977$

or

$\bar{m}_{M}=\left(\frac{7.7857}{\sqrt{2 E}}-1.32977\right)\left(\frac{M}{C}+\frac{1}{2}\right)^{1 / 2}$

The coefficient of determination $R^{2}=0.95876$.

The error between the experimental and calculated values of $k$ and $b$ may be due to the average fragment quality calculated by the software and the detonation velocity calculated from the formula.

In conclusion, the relationship between the average fragment quality $\bar{m}_{M}$ and the energy $E$ released by an explosive is 


$$
\bar{m}_{M}=\left(\frac{k}{\sqrt{2 E}}+b\right)\left(\frac{M}{C}+\frac{1}{2}\right)^{1 / 2}
$$

where $k$ and $b$ are constants related to the type of explosive.

The method of using the average fragment quality $\bar{m}_{M}$ to evaluate the released energy $E$ of a warhead after initiation is as follows:

(1) Measure or calculate the detonation velocity $D_{e}(\mathrm{~km} / \mathrm{s})$ of explosives with different densities. An ion probe can be used to measure $D_{e}$, and equation (10) or (11) can be used to calculate $D_{e}$.

(2) Calculate the shell mass $M(\mathrm{~g})$.

(3) Using the charge density $\rho_{e}$ in step (1), calculate the charge mass $C$ (g).

(4) Calculate the average quality $\bar{m}_{M}$ of fragments produced by complete detonation of explosives with different charge densities using a recovery experiment for a static explosion in a well or appropriate software.

(5) Calculate $\bar{m}_{M} /\left[\left(\frac{M}{C}+\frac{1}{2}\right)^{1 / 2}\right]$ (g) using the results obtained in steps (2)- (4).

(6) Use the result of step (1) to calculate $\frac{1}{0.338 D_{e}}(\mathrm{~s} / \mathrm{km})$.

(7) Use the results of steps (5) and (6) to plot the relationship between $\bar{m}_{M} /\left[\left(\frac{M}{C}+\frac{1}{2}\right)^{1 / 2}\right]$ and $\frac{1}{0.338 D_{e}}$, fit a linear equation, and obtain relationships similar to equations (14) and (18).

\section{Conclusions}

We derived the relationship between the average quality $\bar{m}_{M}$ of fragments formed after warhead initiation and the energy $E$ released by an explosive. The Gurney formula was used to establish this relationship through the initial fragment velocity $v_{0}$. The equation of this relationship is:

$$
\bar{m}_{M}=\left(\frac{k}{\sqrt{2 E}}+b\right)\left(\frac{M}{C}+\frac{1}{2}\right)^{1 / 2}
$$

The coefficients $k$ and $b$ in the relation can be obtained by corresponding calculation or by experiment. These coefficients were found to be related to the type of explosive. The equation can be used to study the conversion of detonation damage energy under different explosive properties and different densities of the same explosive. It provides a theoretical basis for optimizing efficiency of charge energy use in high-energy conventional damage technology and warhead design, and provides a quantitative method for evaluating insensitive ammunition.

\section{Acknowledgment}

We thank A.P. Yu Zheng, Dr. Dou Hong, from School of Mechanical Engineering, Nanjing University of Science and Technology, for their suggestions on this paper.

Author's Contributions: Methodology, Wenbin Li; Writing - original draft, Yiming Li; Writing - review \& editing, Yiming Li and Wenbin Li; Experiment, Junhao Liu, Bo Pu and Qing Zhang; Supervision, Xiaoming Wang.

Editor: Marcílio Alves.

\section{References}

Mott, N. F., Linfoot, E. H., (1943). A theory of fragmentation of shells and bombs. Report AC3348, U.K. Ministry of Supply.

Mott, N. F., (1947). Fragmentation of shell cases. Proceedings of the Royal Society A, 189:300-308. 
Grisaro H., Dancygier A. N., (2016). On the problem of bare-to-cased charge equivalency. Int J Impact Eng 94:13-22.

Held M., (1990). Fragment mass distribution of HE projectiles. Propellants, Explosives. Pyrotechnics 15:254-260.

Smirnov E. B., Kostitsin O. V., Koval A. V., et al. (2016). Model of non-ideal detonation of condensed high explosives. XXXI International Conference on Equations of State for Matter (ELBRUS 2016), Elbrus, Russia, March 1-6.

Shikha Gupta, Nikita Basant, Kunwar Pal Singh, (2015). Identifying high energy molecules and predicting their detonation potency using chemometric modelling approaches. Combustion Theory and Modelling 19:451-464.

P. Song, C. Xiao, F. Shen, et al., (2011). Relationship of Energy Release and Utilization of Non-ideal Detonation of Explosive. Chinese Journal of Explosives \& Propellants 34:44-46.

Gurney R. W., (1943). The initial velocities of fragments from bombs, shells and grenades. Report No 405, Aberdeen proving ground, Ballistic Research Laboratories (BRL), Maryland, USA.

J. J. Zhu, Y. Zheng, W. B. Li, et al., (2019). Axial distribution of fragments from the dynamic explosion fragmentation of metal shells, Int J Impact Eng 123:140-146.

Z. X. Huang, X. D. Zu, (2014). Terminal Effects, Science Press.

Dobratz B. M., Crawford P. C., (1985). LLNL Explosive Handbook Properties of Chemical Explosives and Explosive Simulants. Lawrence Livermore National Laboratory.

J. J. Zhu, Y. Zheng, Y. C. Yang, et al., (2018). Research on the volume and line fractal dimension of fragments from the dynamic explosion fragmentation of metal shells. Powder Technology 331:129-136.

M. J. Zheng, T. C. Yu, Y. L. Zhang, (1990). Explosive Performance and Test Technology, Ordnance Industry Press. 